Check for updates

Cite this: RSC Adv., 2021, 11, 14769

Received 25th January 2021

Accepted 8th April 2021

DOI: $10.1039 / \mathrm{d} 1 \mathrm{ra00652e}$

rsc.li/rsc-advances

\section{Study on the enhancing water collection efficiency of cactus- and beetle-like biomimetic structure using UV-induced controllable diffusion method and 3D printing technology $\dagger$}

\author{
Linhui Peng, (DD Keqiu Chen, \$ Deyi Chen, Jingzhi Chen, Jie Tang, Shijie Xiang, \\ Weijiang Chen, Pengyi Liu, ${ }^{*}$ Feipeng Zheng* and Jifu Shi*
}

\begin{abstract}
Collecting water from fog flow has emerged as a promising strategy for the relief of water shortage problems. Herein, using a UV-induced (ultraviolet light induced) controllable diffusion method combined with technology of three-dimensional (3D) printing, we fabricate biomimetic materials incorporating beetle-like hydrophobic-hydrophilic character and cactus-like cone arrays with various structure parameters, and then systematically study their fog-harvesting performance. The UV-induced controllable diffusion method can break away from the photomask to regulate the hybrid wettability. Moreover, employing 3D printing technology can flexibly control the structure parameters to improve the water collection efficiency. It is found that the water collection rate (WCR) can be optimized by controlling the hybrid wettability of the sample surface and cone distance and using substrates with printed holes, which lead to a $109 \%$ increase of WCR.
\end{abstract}

\section{Introduction}

It is known that water shortages are becoming increasingly serious, as almost two-thirds of the lands on Earth are short of water. ${ }^{1,2}$ Fog and moisture in the atmosphere are very promising resources, as they account for $10 \%$ of the pure water in the world. ${ }^{3}$ Therefore, the design of efficient water-collecting structures is strongly expected. Through natural selection, some plants and animals such as cacti and beetles have evolved the ability to efficiently collect water from the fog by taking advantage of the Laplace pressure gradien $\mathrm{t}^{4-6}$ and hydrophobichydrophilic character, ${ }^{7-10}$ respectively. Hence, it is expected that building a structure combining beetle-like hydrophobichydrophilic character ${ }^{11-15}$ and cactus-like cones ${ }^{16,17}$ can further promote the water collection efficiency, which will be higher than either of theirs. ${ }^{18}$

To achieve the efficient water collection, it is significant to combine beetle-like hydrophobic-hydrophilic character and cactus-like cones. The hybrid hydrophobic-hydrophilic wettability shows better performance in water collection than the uniformly wettability such as superhydrophobic and

Siyuan Laboratory, Guangzhou Key Laboratory of Vacuum Coating Technologies and New Energy Materials, Department of Physics, Jinan University, Guangzhou 510632, China. E-mail: tlpy@jnu.edu.cn; fpzheng_phy@email.jnu.edu.cn; shijifu2017@126. com

$\dagger$ Electronic supplementary information (ESI) available: Fig. S1-S8 and Tables S1-S4. See DOI: 10.1039/d1ra00652e

\$ These authors contributed equally. superhydrophilic. ${ }^{11-13}$ Thus, fabricating beetle-like hydrophobic-hydrophilic character is a key factor for efficient water collection. For that numbers of methods such as inkjet printing method, ${ }^{13,19}$ selective modification method ${ }^{20,21}$ and UV-induced method $^{11,22-24}$ have been proposed in previous studies, in which the UV-induced method is one of the most practical techniques, but most of them inevitably need photomasks. The photomask makes the fabrication process complex, increases the cost and limits the manufacture at a large scale. To break away from the additional photomasks, some related researches appeared. ${ }^{9,25,26}$ In our previous work, we presented a maskless UV-induced method to make the superhydrophobic-superhydrophilic character. ${ }^{26}$ Its principle is that under UV irradiation, the hydrophobic adhesive below $\mathrm{TiO}_{2}$ film can decompose into small molecules with low surface tension, and transfer to the $\mathrm{TiO}_{2}$ surface due to the surface tension. Thus, the regions coated with small molecules converts to superhydrophobic and other regions still remain superhydrophilic for long time (Table S4†). Inspired by this work, we further improved the method to meet the requirements of water collection in this work. Here, we creatively introduce the mixing of the hydrophobic and hydrophilic (HB-HL) adhesives to quantitatively control wettability. Therefore, in terms of manufacturing beetle-like hydrophobichydrophilic character, this method has good application in water collection.

Fabricating cactus-like structure is another important factor for high water collection efficiency. Electrochemical etching methods, ${ }^{27-29}$ mold-making methods ${ }^{30}$ and laser direct 
methods ${ }^{31,32}$ are usually applied to build the cactus-like structure. Meanwhile, as an additive manufacturing technology, 3D printing technology possesses the advantages of manufacturing complex structure and individual customization, which has made many remarkable achievements in the field of bionics. ${ }^{33-35}$ Particularly, compared with traditional methods, it is flexible and convenient to produce water collecting structures with different morphologies for structure optimization, which gets rid of the restrictions of mold and other complex production process. In addition to the morphology of the cones, ${ }^{36,37}$ considering the water capture and transportation, other structural parameters such as cone distance and morphology of substrate also affect water collection efficiency. Thus, we adopted the 3D printing technology to produce water collection device with different structural parameters, and explored the effect of cone distance and the holes on the substrate, which are significant to improve the water collection efficiency.

In this paper, a simple UV-induced controllable diffusion method and 3D printing technology are utilized to create a series of high-quality biomimetic materials with different superhydrophobic-superhydrophilic characters and structure parameters, which are composed of beetle-like character and cactus-like cone array. By adjusting superhydrophobic-superhydrophilic character and structural parameters, the water collection rate (WCR) can raise $109 \%$ to reach $2.68 \mathrm{~g} \mathrm{~h}^{-1} \mathrm{~cm}^{-2}$, realizing greater optimization. ${ }^{38,39}$

\section{Experimental}

\subsection{Materials}

Commercial hydrophilic adhesive (Beierte, China), organosilicon adhesive (99\%) (YMJ, Delta, China), titanium dioxide (nano-TiO 2 , P25) (T823119, Macklin, China), ethanol, deionized water, photopolymer (SLAY-2, Liantai, China).

\subsection{The fabrication of the samples}

First, various densities of cone arrays (with cone distances of 1 , $1.5,2,2.5,3 \mathrm{~mm}$ ) on $2 \mathrm{~cm} \times 2 \mathrm{~cm}$ substrates with and without holes were printed using SLA 3D printer (LT600, Lizhu, China), respectively (inset of Fig. 1a). The diameter and height of a single cone in the arrays are $1 \mathrm{~mm}$ and $3 \mathrm{~mm}$, respectively. Then hydrophobic adhesive (silicone adhesive) and hydrophilic adhesive (commercial acrylic adhesive) were fully mixed with different mass ratios $(1: 0,1: 9,3: 7,1: 1,7: 3,9: 1)$ and then coated on the substrates, respectively. The $\mathrm{TiO}_{2}$ alcohol dispersion was sprayed $\left(1 \mathrm{~g} \mathrm{TiO}_{2}\right.$ was dispersed in $75 \mathrm{~mL}$ anhydrous alcohol) on the substrates as followed (Fig. S1 $\dagger$ ). Finally, the sample was subjected to ultraviolet-light (UV) irradiation for 3 hours (two $8 \mathrm{~W}$ UV lamps were used, which were $5 \mathrm{~cm}$ away from the samples) to obtain the final samples.

\subsection{Experiments on the water-collecting ability of samples}

The samples were hung vertically on the iron platform with clamps. And the droplets were collected in a container below the sample. The relative humidity and temperature of the environment were maintained within $70-80 \%$ and $25.0 \pm 1{ }^{\circ} \mathrm{C}$,

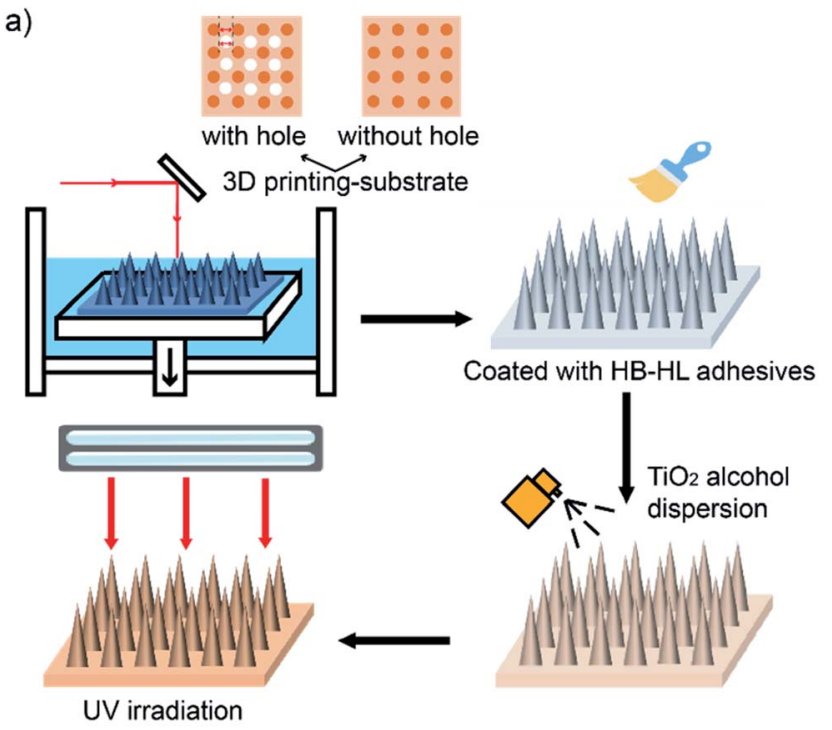

b)

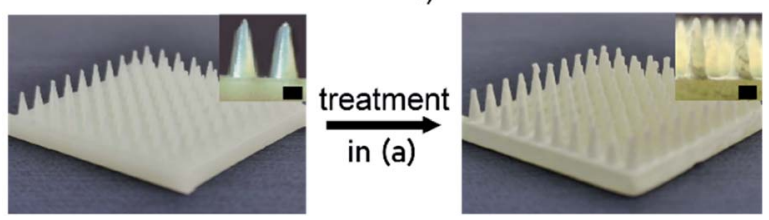

Fig. 1 (a) Schematic diagram of the fabrication of cone arrays with superhydrophobic-superhydrophilic character. Panels (b) and (c) display the photos of the samples before and after the fabricating process as shown in panel (a), respectively. Insets: magnifications around the cones. The scale bar for the inset images is $1 \mathrm{~mm}$.

respectively. The fog was generated by a commercial ultrasonic humidifier (h-010, BlueLounge, China). The actual fog volume was $600 \mathrm{~mL} \mathrm{~h}^{-1}$, and the fog velocity was approximately $10 \mathrm{~cm}$ $\mathrm{s}^{-1}$. The outlet with diameter of $5 \mathrm{~cm}$ was $8 \mathrm{~cm}$ away from the sample. The volume of the collected water was recorded every 30 minutes for a two-hour cycle. We strictly adopted the variablecontrolling approach to optimize the parameters of cone distance, HB-HL adhesive ratio and morphology of the substrate at the same time to obtain the best water collection efficiency by involving the synergy effect of them. For examples, when studying the influence of different HB-HL adhesive ratios, we fixed the cone distance $(2 \mathrm{~mm})$ and the morphology of the substrate (with/without holes), and only changed the HB-HL adhesive ratio; when studying the effect of cone distance on water collection efficiency, we fixed the ratio of HB-HL adhesive (1:1) and the substrate morphology (with/without holes), and only changed the cone distance.

\subsection{Characterization}

Scanning electron microscopy (SEM) images were taken by the scanning electron microscope (Hitachi S3700N, Japan). The contact angle data were obtained on a commercial contact angle system (JC2000C2, Powereach, China) at ambient temperature using a $5 \mathrm{~mL}$ droplet as the indicator. The material of the samples was analyzed by Fourier transform infrared 
spectrometer (FTIR) (Thermo Scientific Nicolet iS20, USA). X-ray photoelectron spectroscopy (XPS) measurements were performed by an AXISNOVA instrument (Thermo Fisher Scientific, K-ALPHA+, USA) using a monochromatic Al K $\alpha$ X-ray source $(1486.68 \mathrm{eV})$. Digital images and videos were captured using a CCD camera with $10 \mathrm{M}$ Micro Lens (Vitken, SHL-500 W-NEW, China) and high-speed camera (Phantom Mro311, USA).

\section{Results and discussion}

\subsection{Fabricating water collection materials by $3 \mathrm{D}$ printing and UV-induced controllable diffusion method}

Fig. 1a shows the fabricating process of cactus-like spines with beetle-like superhydrophobic-superhydrophilic character. First, we used 3D printing technique to construct cone arrays substrates, which can be found in Fig. 1b. Then, the substrates were coated by the mixture of different mass ratio of HB-HL adhesives, respectively. Furthermore, the surface of the obtained base was sprayed with $\mathrm{TiO}_{2}$ alcohol dispersion and then exposed to UV light to obtain final samples, which were shown in Fig. 1c.

In the course of creating the above superhydrophobicsuperhydrophilic character, we adopted a simple UV-induced controllable diffusion method by mixing HB-HL adhesives
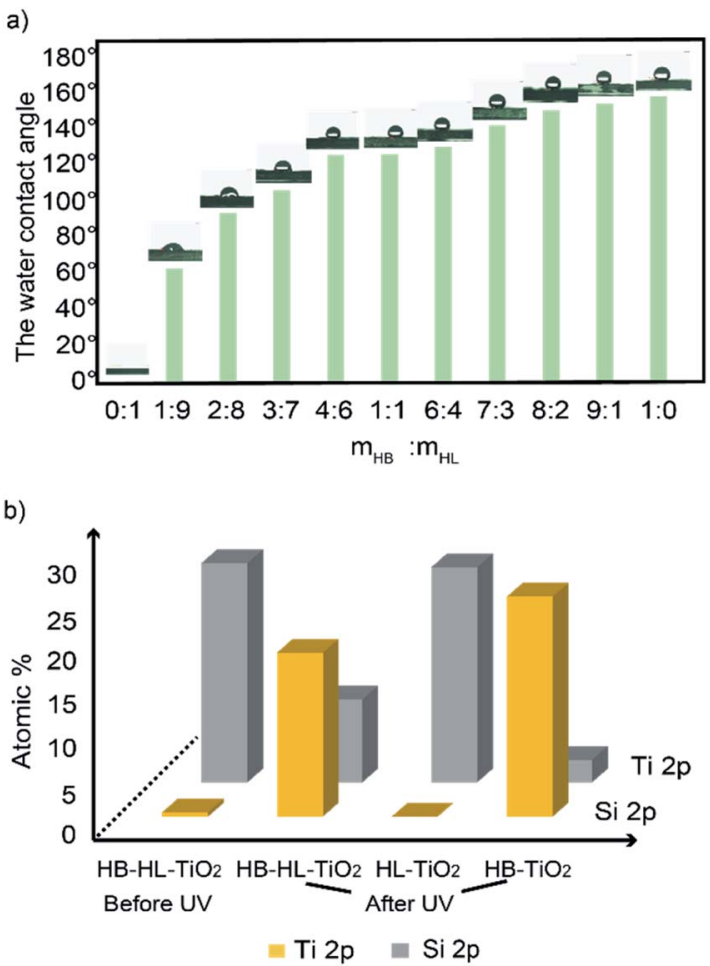

Fig. 2 (a) Evolutions of the water contact angles with respect to the ratio of masses between hydrophobic $\left(m_{\mathrm{HB}}\right)$ and hydrophilic $\left(m_{\mathrm{HL}}\right)$ adhesives on samples after UV irradiation. (b) The atomic proportional of $\mathrm{Si}$ and $\mathrm{Ti}$ element in $\mathrm{HB}-\mathrm{HL}-\mathrm{TiO}_{2}$ sample before and after UV irradiation and the samples $\left(\mathrm{HL}-\mathrm{TiO}_{2}, \mathrm{HB}-\mathrm{HL}-\mathrm{TiO}_{2}\right.$ and $\left.\mathrm{HB}-\mathrm{TiO}_{2}\right)$ after UV irradiation measured by XPS. The CA measurements were conducted on the flat substrate at room temperature. All of the water droplet volumes in these images were $5 \mu \mathrm{L}$. with different mass ratios as mentioned above. Fig. 2 displays the water contact angles of the final samples with different mass ratios of adhesives after UV irradiation. It is found that the water contact angles, which are positively correlated to hydrophobicity of a surface, go up as the increase of the mass ratio. We also show that the character can be regulated by UV exposed time in Fig. S4. $\dagger$ The above result indicates that our method is feasible and economical, as it can effectively tune superhydrophobic-superhydrophilic character of sample without need of an additional mask as used in previous studies. ${ }^{11,22,40}$

Then, it is nature to ask how the coated adhesives modify the hybrid wettability of the sample after UV irradiation as shown in Fig. 2a. We first perform scanning electron microscopy (SEM) measurement to examine the surface topography of the samples with different mass ratios before and after UV irradiation. We selected and studied the three representative samples with the mass ratio of $0: 1,1: 1$ and $1: 0$, whose SEM images are shown in Fig. 3. Before UV irradiation, there is no significant difference in the samples with different mass ratio of HB-HL adhesives, (Fig. 3a, c and e) which are composed of the accumulated large particles formed by $\mathrm{TiO}_{2}$ aggregation basically, (Fig. $\mathrm{S} 1 \dagger$ ) leading to the formation of rough surface contributed by large number of air pockets. After UV irradiation, the change on the sample without hydrophobic adhesive is negligible (Fig. 3b). However, for the samples with hydrophobic adhesive, (Fig. 3d and f) the air pockets are filled by upward colloidal substances induced by the UV irradiation, leading to the compaction of the a)

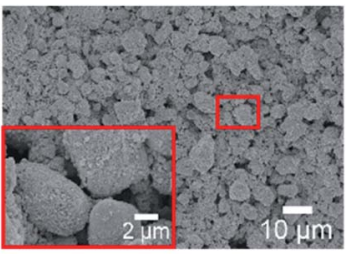

c)

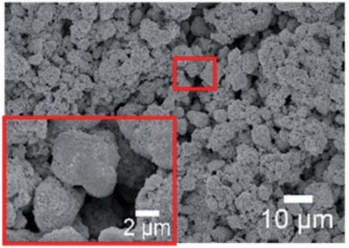

e)

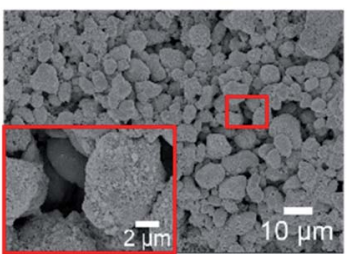

)

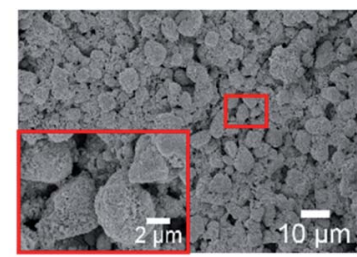

d)

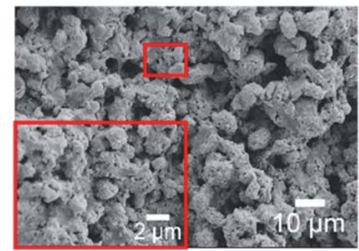

f)

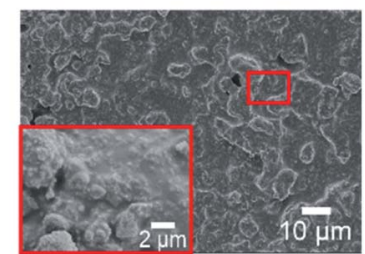

Fig. 3 SEM images of the samples prepared on the flat substrate before and after UV irradiation with different adhesives ratio. ( $a$ and $b$ ) The samples with $0: 1$ mass ratio of $\mathrm{HB}-\mathrm{HL}$ adhesives before and after UV irradiation, respectively, ( $c$ and $d$ ) the samples with $1: 1$ mass ratio of $\mathrm{HB}-\mathrm{HL}$ adhesives before and after UV irradiation, respectively (e and f) the samples with 1:0 mass ratio of $\mathrm{HB}-\mathrm{HL}$ adhesives before and after UV irradiation, respectively. The magnifications of the regions enclosed by red rectangle boxes are also shown. 
surface. By closer inspection, it is found that the larger ratio of the hydrophobic adhesive of the sample, the more compact of its surface, where more upward colloidal substances are found (comparing Fig. 3b, d and f). In the following part, we further confirm that the upward colloidal substances are owned by organosilicon adhesive by XPS and FTIR measurements.

Fig. 4a displays high-resolution XPS spectra for $\mathrm{O}$ 1s for the following five samples: $\mathrm{HB}$ adhesive, $\mathrm{HL}$ adhesive, $\mathrm{HB}-\mathrm{HL}$ adhesives (the mass ratio is $1: 1$ ), samples with the $\mathrm{HB}-\mathrm{HL}$ adhesives before and after UV irradiation. The dominant feature of the $\mathrm{HB}$ adhesive is a peak centered at $532.1 \mathrm{eV}$, which can be assigned to O-Si. Similarly, the peaks of HL adhesive at 533.2, $531.8 \mathrm{eV}$ are associated with $\mathrm{O}=\mathrm{C}$, O-C, respectively. ${ }^{41} \mathrm{By}$ comparing the above two curves with that of the HB-HL adhesives, one can find that the peaks in the latter curve can be regarded as the superposition of the peaks belonging to $\mathrm{HB}$ and $\mathrm{HL}$ adhesives, with slightly blue-shifted peaks of $\mathrm{O}-\mathrm{Si}$ and $\mathrm{O}=\mathrm{C}$ by $0.3 \mathrm{eV}$ and $0.2 \mathrm{eV}$, respectively, which is the result of the interaction between HB and HL adhesives after they are mixed. The FTIR measurement also supports the above conclusion, as all the FTIR peaks of HB-HL adhesives can be traced back to the ones of HB or HL adhesives as discussed below. ${ }^{26,42}$ As shown in Fig. 4c, the peaks of HB (black curve) centered at 2962.1, 1256.4, 1051.9-1009.5 and $788.7 \mathrm{~cm}^{-1}$ are related to the stretching vibrations of $\mathrm{C}-\mathrm{H}, \mathrm{CH}_{3}, \mathrm{Si}-\mathrm{O}$ and bending vibration of $\mathrm{C}-\mathrm{H}$, respectively. ${ }^{42}$ The peaks of HL (red curve) centered at 2956.3, 1726.4, 1151.7 and $696.7 \mathrm{~cm}^{-1}$, are assigned to the stretching vibration of $\mathrm{O}-\mathrm{H}, \mathrm{C}=\mathrm{O}, \mathrm{C}-\mathrm{O}$ and the bending vibration of $\mathrm{C}-\mathrm{H}$, respectively. By comparing the peaks of $\mathrm{HB}-\mathrm{HL}$ (green curve) with the ones of $\mathrm{HB}$ and $\mathrm{HL}$, one can find that the peaks of $\mathrm{HB}-$ HL adhesives centered at 2958.7, 1727.4, 1256.8, 1052.9-1011.4, 791.6 and $697.6 \mathrm{~cm}^{-1}$, can be assigned to the compositions of $\mathrm{C}-\mathrm{H}$ owned by $\mathrm{HB}$ adhesive and $\mathrm{O}-\mathrm{H}$ owned by $\mathrm{HL}$ adhesive, $\mathrm{C}=\mathrm{O}$ of $\mathrm{HL}$ adhesive, $\mathrm{CH}_{3}$ of $\mathrm{HB}$ adhesive, the combination of $\mathrm{C}-\mathrm{O}$ owned by $\mathrm{HL}$ adhesive and $\mathrm{Si}-\mathrm{O}$ owned by $\mathrm{HB}$ adhesive, $\mathrm{C}-\mathrm{H}$ owned by $\mathrm{HB}$ adhesive as well as $\mathrm{C}-\mathrm{H}$ owned by $\mathrm{HL}$ adhesive, respectively. Furthermore, the peaks of $\mathrm{HB}-\mathrm{HL}$ adhesives generally exhibit slight blue shift, which is consistent to the XPS results that the interaction between $\mathrm{HB}$ and $\mathrm{HL}$ adhesives lead to the slightly blue-shift. After covered by the mixed adhesives, the substrate was further coated by $\mathrm{TiO}_{2}$ before UV irradiation as mentioned before, whose XPS spectrum is dominated by a peak associated with O-Ti (filled by yellow in Fig. 4a). ${ }^{43}$ By comparison, the spectrum after UV irradiation exhibits an additional peak centered at $532.7 \mathrm{eV}$, which is assigned to $\mathrm{O}-\mathrm{Si}$ belonging to $\mathrm{HB}$ adhesive, but with a blue shift of $0.6 \mathrm{eV}$ due to the weak interaction between the HB-HL adhesives and titanium dioxide. Moreover, comparing the broadband scan spectra of the samples before and after UV irradiation shown in Fig. $4 \mathrm{~b}$ and Table $\mathrm{S} 1, \dagger$ one can find that the atomic proportion of Ti decreases, accompanied by the emergence of Si 2p peaks after UV irradiation. The above observations demonstrate that only small molecules, belonging to $\mathrm{HB}$ adhesive, spreads upward to the surface and covers the $\mathrm{TiO}_{2}$ due to UV irradiation. It should be mentioned that the upward of the small molecules are driven by the surface tension as discussion in previous works. ${ }^{26,44}$ Our FTIR data also exhibit consistent
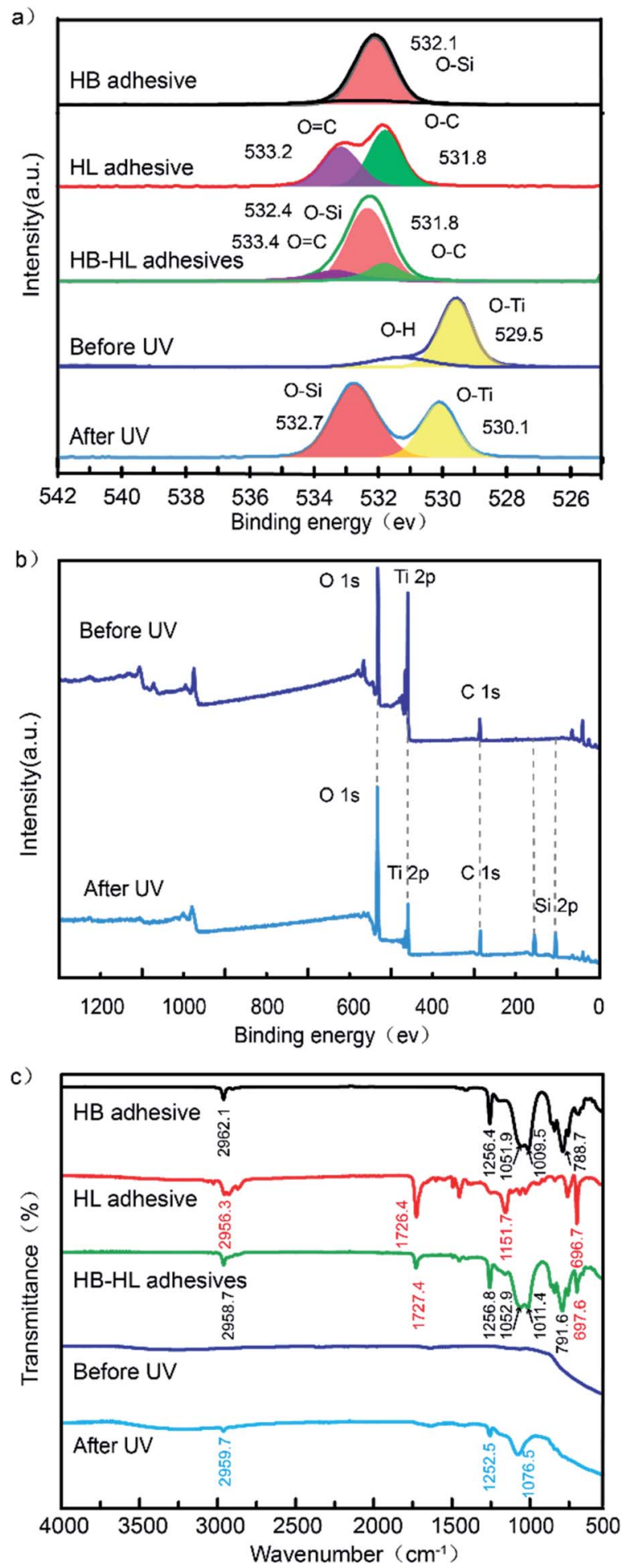

Fig. 4 (a) High-resolution XPS spectra for $\mathrm{O}$ 1s of $\mathrm{HB}$ adhesive, $\mathrm{HL}$ adhesive, $\mathrm{HB}-\mathrm{HL}$ adhesives (the mixed ratio is $1: 1$ ), the sample with the mixed adhesive before and after UV irradiation, respectively. (b) Broadband scan XPS spectra of the samples before and after UV irradiation. (c) FTIR spectra of the five samples in (a).

result. As shown in Fig. 4c, there was no obvious peak in range of $1000-4000 \mathrm{~cm}^{-1}$ before UV irradiation. In contrast, only the characteristic peaks of $\mathrm{HB}$ centered at 2959.7, 1252.5 and 
$1076.5 \mathrm{~cm}^{-1}$ corresponding $\mathrm{C}-\mathrm{H}, \mathrm{CH}_{3}$ and $\mathrm{Si}-\mathrm{O}$ emerge after UV irradiation (blue curve), confirming the upward small molecules belonging to HB adhesive. The upward small molecules reduce the surface energy where it locates, transforming the character into superhydrophobic, which is originally superhydrophilic due to high surface energy of $\mathrm{TiO}_{2}$. Therefore, the hybrid wettability of the surface is modified.

Since Si element only exists in the HB adhesive (Fig. S2†), we can assess the distribution of superhydrophobic region on the hybrid wettability surface via Si element distribution (Fig. S3†). Furthermore, by examining the atomic proportional of $\mathrm{Si}$ element in the samples with different HB ratios as shown in Fig. 2b (more details are tabulated in Table S1†), we find that the amounts of Si upward after UV are proportional to the ratio of HB. This is also responsible for the result in Fig. 2a that the water contact angles of the samples are positive correlated to the ratio of $\mathrm{HB}$ adhesive on the surface. Therefore, we have shown that the mixing of HB-HL adhesives is an effective way to control the wettability without need of a photomask. In the following part, we will show that the hybrid HB-HL structure have good performance in water collection.

\subsection{Fog-harvesting ability}

Water collection tests were performed to explore the influence of different factors of the sample on water collection efficiency, including cone distance, mass ratio between $\mathrm{HB}$ and $\mathrm{HL}$, and hole on substrate. During the test, each sample after UV irradiation with a size of $2 \mathrm{~cm} \times 2 \mathrm{~cm}$ was suspended vertically as shown in Fig. 5c. The relative humidity and temperature of the environment were maintained within $70-80 \%$ and $25.0 \pm 1{ }^{\circ} \mathrm{C}$, respectively. The whole process of water capture can be decomposed as follows: (i) each of the cactus-like cones on the substrate capture droplets from the fog; (ii) driven by Laplace force of the cone and droplets coalescence, the droplets are pushed onto the substrate; (iii) aggregating the droplets as they pass, the merged droplets continually transport down to the bottom of the substrate due to the forces of gravity, during which they are also resisted by viscous force applied by the adjacent cones and substrate; (iv) the accumulated droplet grows increasingly large until its gravity overcome the friction force of the substrate, and then drips down to the container.

3.2.1 The influence of mass ratio of hydrophobic and hydrophilic adhesives on WCR. We first investigate the influence of hybrid wettability on WCR. Fig. 5a displays the WCR of the samples with same cone distance of $2 \mathrm{~mm}$ but different mass ratios between HB-HL adhesives $\left(m_{\mathrm{HB}}: m_{\mathrm{HL}}\right)$. We first focus on the data without hole on the substrate (the data with hole will be discussed later). The WCR is $1.93 \mathrm{~g} \mathrm{~h}^{-1} \mathrm{~cm}^{-2}$ when the ratio is $1: 0$. Then it first increases as the ratio of $\mathrm{HL}$ adhesive, and reaches the maximum value of $2.46 \mathrm{~g} \mathrm{~h}^{-1} \mathrm{~cm}^{-2}$ when the ratio is $1: 1$. This is because the increase of HL adhesive will facilitate the droplet transportation on the cone surface, which is referred to the aforementioned step (ii). The facilitation is due to the fact that the more hydrophilic of a cone surface, the larger contact area between the droplet and the

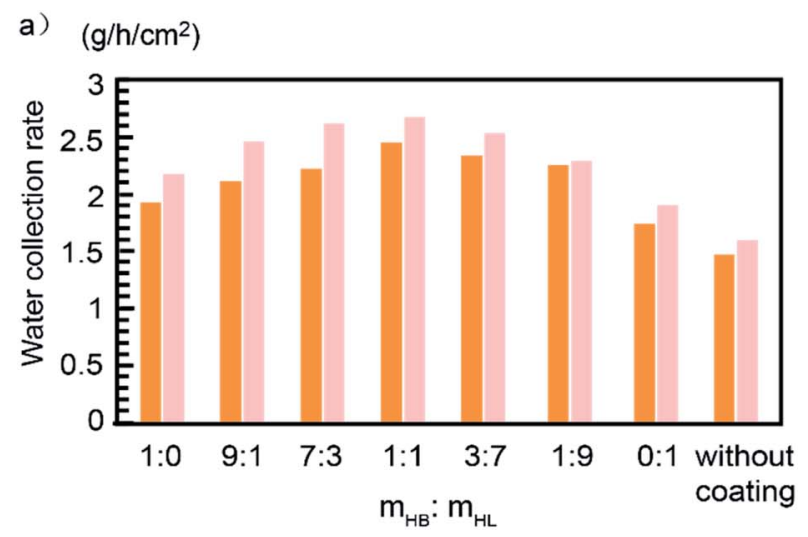

with holes without hole

b)

$$
\Delta P=-\int_{r 1}^{r 2} \frac{2 \gamma}{\left(r+R_{0}\right)^{2}} \sin \beta d z
$$

c)
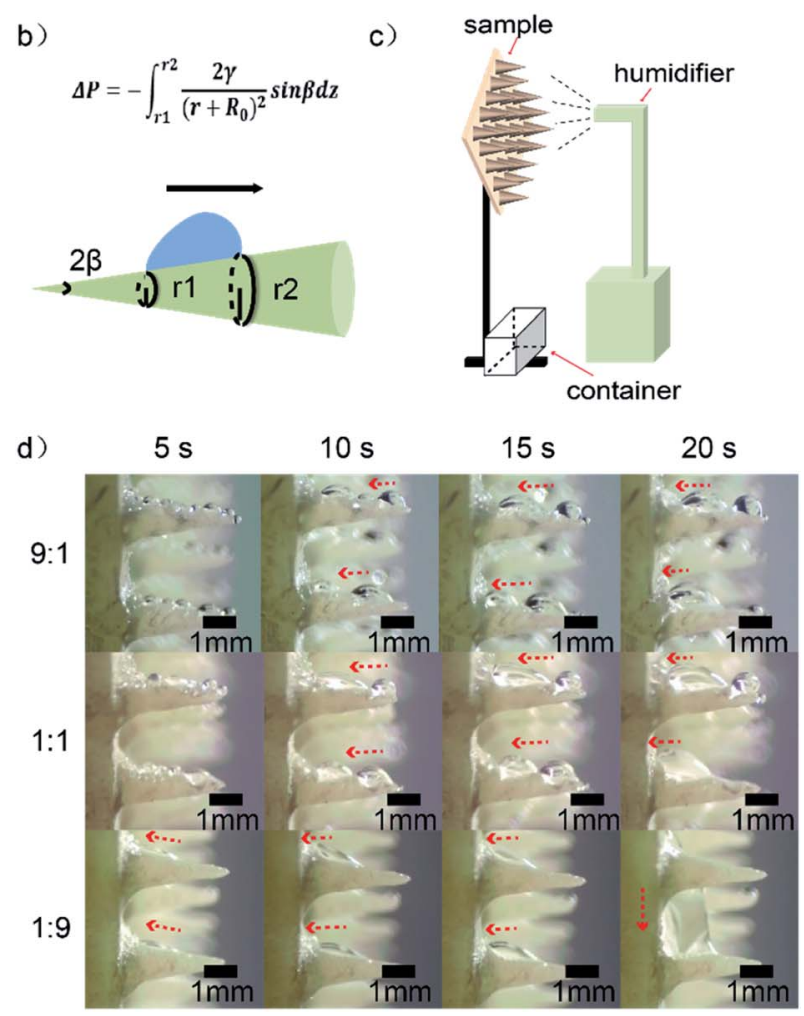

Fig. 5 (a) WCRs of the samples coated by $\mathrm{HB}-\mathrm{HL}$ adhesives $\left(m_{\mathrm{HB}^{-}}\right.$ $: m_{\mathrm{HL}}$ ) with different mass ratios, as well as a sample without coating. The cone distances of all the samples are $2 \mathrm{~mm}$. (b) Schematic illustration of Laplace force $(\Delta P)$ of a droplet on the conical surface. (c) Experimental setup for the collection of water from fog. (d) Side view of photographs of the samples magnified around specific cones at the times of $5,10,15$ and $20 \mathrm{~s}$.

surface (see Fig. 2, 5d and S5†), ${ }^{4}$ leading to larger Laplace force according to the following equation: ${ }^{45}$

$$
\Delta P=-\int_{r_{1}}^{r_{2}} \frac{2 \gamma}{\left(r+R_{0}\right)^{2}} \sin \beta \mathrm{d} z,
$$

where $\gamma$ is the surface tension of water, $r_{1}$ and $r_{2}$ are the local radii of the cone at the two opposite sides of the droplet (see Fig. $5 \mathrm{~b}$ ), $R_{0}$ is the radius of the droplet and $\mathrm{d} z$ is along the direction connecting the origins of the local circles with their 
radiuses $r_{1}$ and $r_{2}$ as shown in Fig. $5 \mathrm{~b}$. The larger Laplace force accelerates the droplet transportation on the cone, leading to higher WCR as we have seen in the Fig. 5a. However, it is also seen that further increasing the ratio of hydrophilic adhesive, the WCR tends to decrease. This can be understood as follows. Employing excess hydrophilic adhesive, droplets tend to spread out and form a thin water-film on the surface, leading to high viscous force on the substrate, which weakening the ability of water transportation in the step (iii). ${ }^{46}$ The formation of the water-film in the surface with excess hydrophilic adhesive is indeed found in our experiment, which can be seen in the sample with the ratio $1: 9$ at the time of $20 \mathrm{~s}$ shown in Fig. $5 \mathrm{~d}$. Therefore, the increase of hydrophilic adhesive facilitates the droplet transportation from the cone surface to the substrate [step (ii)], but excess hydrophilic adhesive increases viscous force, which is against droplet transportation on the substrate [step (iii)]. In our experiment, the WCR is optimal when the mass ratio of $\mathrm{HB}-\mathrm{HL}$ adhesives is $1: 1$ (also see Fig. $\mathrm{S} 7 \dagger$ ). A reasonable mass ratio of $\mathrm{HB}-\mathrm{HL}$ adhesives should be determined for the design of similar water-collecting materials to obtain high WCR.

It should be noted that the mixing of HB-HL adhesives has at least the two functionals: (1) adjusting the overall surface wettability to moderate wettability ${ }^{47}$ for water collection; (2) taking advantage of fog capture ability of the HL region and water transportation ability of the HB region, which enhance the fog-capture and droplet transportation capacity on the substrate. Therefore, though the overall surface wettability for a droplet on the HB-HL structures can only be HL or HB, this structure can have higher water collecting rate than the ones with similar wettability but without mixing adhesives as we shown in Fig. S9. $\dagger$

3.2.2 The influence of cone distance on WCR. Significantly, we found that the cone distance can strongly affect the WCR. The substrates with different cone spacing $(1,1.5,2,2.5$ and 3 $\mathrm{mm}$ ) were fabricated using $3 \mathrm{D}$ printing technology. The mass ratio of HB-HL adhesives is kept at $1: 1$. The WCRs for the above samples are displayed in Fig. 6a. It is seen that when cone distance rises from 1 to $2 \mathrm{~mm}$, the WCR is nearly doubled by increasing from $1.28 \mathrm{~g} \mathrm{~h}^{-1} \mathrm{~cm}^{-2}$ to the maximum value of $2.46 \mathrm{~g}$ $\mathrm{h}^{-1} \mathrm{~cm}^{-2}$. Further increasing cone distance will decrease WCR. The above observation indicates that cone distance has a strong impact on WCR of the sample. To understand how the cone distance affects the WCR of the sample, we take photos (Fig. 6b and $\mathrm{S}^{\dagger}+$ ) of the water-collecting process of different samples for comparison. Fig. $6 \mathrm{~b}$ displays the photos of the samples with cone distances of 1, 2 and $3 \mathrm{~mm}$, which will be referred to overdense, medium-dense and over-sparse samples, respectively. We can see that a sizable droplet (enclosed by a red circle) appears at the bottom of the over-sparse sample at the $90 \mathrm{~s}$. In contrast, there is no obvious droplet accumulation in the bottom of other two samples at the same time, indicating the highest transportation efficiency of the over-sparse sample. This is because the viscous force of the cone hinders droplet transportation on the substrate. Namely, the sparser the cone array, the smaller resistance force the droplets are subjected to, leading to higher transportation efficiency on the substrate in
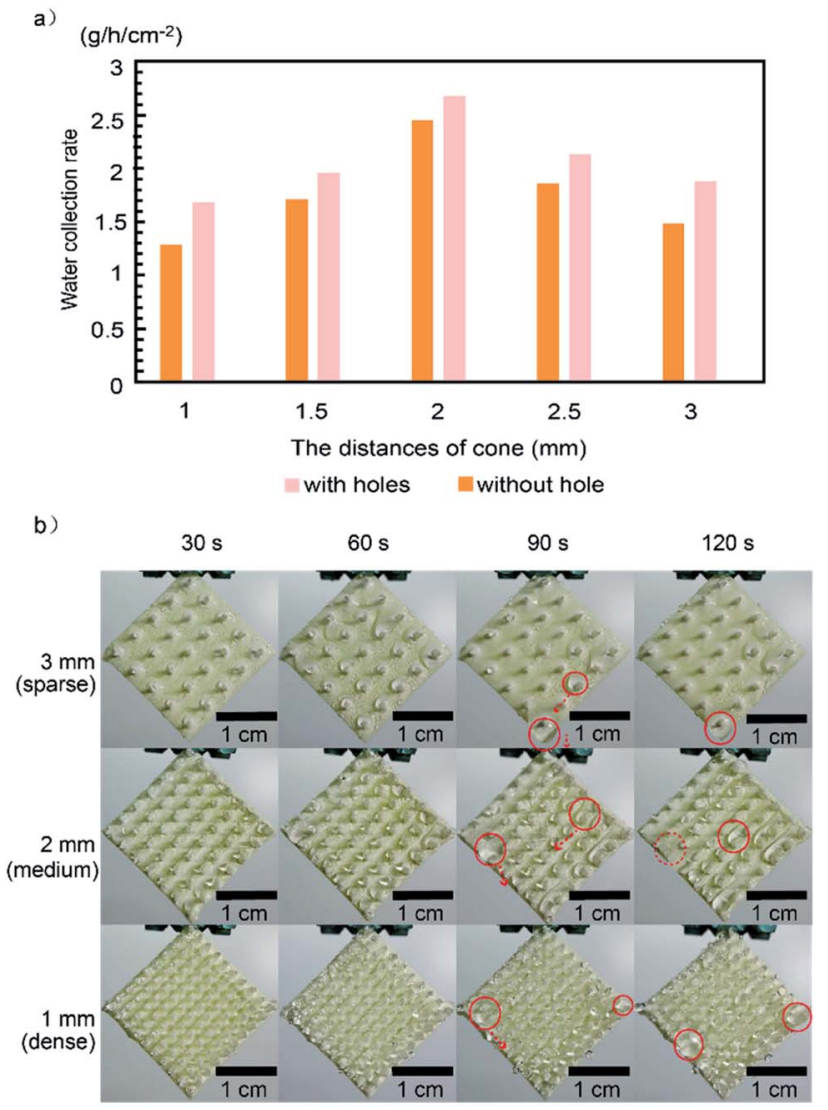

Fig. 6 (a) Fog collection efficiency of the samples with different cones distances. (b) Photographs of the cone arrays with different cones distances during water collection within 2 min. The mass ratio of HB$\mathrm{HL}$ adhesives was 1:1 for this experiment. The sizable droplet and detached droplet are enclosed by red solid and dotted circle, respectively.

the step (iii). However, the highest transportation efficiency does not mean the highest WCR, since reducing cone density also reduces the areas of water capture, leading to sparse droplet on the sample. This is consistent with that though the droplet transportation in over-sparse sample is fast, the number of captured droplets in the step (i) is small, which is against high WCR. Furthermore, some sizable droplets (red circle) can be seen in each case of the over- and medium-dense samples at the $90 \mathrm{~s}$ (see Fig. 6b). At the time of $120 \mathrm{~s}$, a droplet in the medium-dense sample has already broken away from sample and dripped down to the container. By contrast, droplets of the over-dense sample are still hanging on the sample at the same time. The different behaviours of droplet transportation in the over- and medium-dense samples can be understood similarly. The denser of the cones, the larger viscous force the droplets are subjected to, which reduces the transportation efficiency of the droplets in the step (iii). Therefore, over-sparse and over-dense cone arrays are not beneficial to efficient water collection. It is of particularly significance to choose the eligible cone distance, which makes a balance between the transportation efficiency in the step (iii) and the number of captured droplets in the step (i), 
to achieve high WCR. In our work, the sample with cone distance of $2 \mathrm{~mm}$ is the eligible one.

3.2.3 The influence of holes on substrate on WCR. Apart from the mass ratio of $\mathrm{HB}-\mathrm{HL}$ adhesives and cone distance, we find that the presence of holes on the substrate can promote WCR. By comparing the WCR of the samples with/without the 3D-printed holes on the substrate shown in Fig. 5a and 6a, it is interesting to see that the samples with holes generally exhibit higher WCR. For example, under the equivalent conditions that the cone distance is $2 \mathrm{~mm}$ and the mass ratio is $1: 1$, the WCR is 2.46 and $2.68 \mathrm{~g} \mathrm{~h}^{-1} \mathrm{~cm}^{-2}$ for the sample without and with holes, respectively. This is somewhat confusing, since the sample without hole possesses additional water-capture region on the substrate and are supposed to enjoy higher WCR intuitively. To clarify the above confusing, we additionally prepare two kinds of the samples with and without holes printed by 3D printing technology, both of which are without cone arrays and coated by HB-HL adhesives with the ratio $1: 1$. As shown in Fig. 7a and b, we took photos and recorded the time of the moments that the first five droplets started to detach from the substrates for the above two samples, respectively. It is found that the time for the samples with and without holes take to drip the five droplets are 133.0 and $171.6 \mathrm{~s}$, and the averaged mass per droplet are 0.041 and $0.048 \mathrm{~g}$, leading to the WCR of 0.845 and $0.763 \mathrm{~g} \mathrm{~h}^{-1} \mathrm{~cm}^{-2}$, respectively (see Tables $\mathrm{S} 2$ and $\mathrm{S} 3 \dagger$ ). And the sample with holes possesses higher WCR due to higher transportation efficiency on the substrate. We note that reducing water-capture regions on the substrate slightly decreases the mass of the dripping droplet in the sample with holes ( $0.041 \mathrm{~g}$ per droplet), however, it significantly facilitates the droplet transportation on the substrate, leading to the higher WCR, which can be understood as follow. As shown in Fig. 7c, each droplet is driven by gravitational force $(G)$ and resisted by viscous force $\left(f_{\mathrm{v}}\right)$ arising from the substrate, which reads: $f_{\mathrm{v}}=\eta S(\mathrm{~d} v / \mathrm{d} y)$, where $\eta$ is the coefficient of viscosity of water, $\mathrm{d} v / \mathrm{d} y$ is the velocity gradient of water, and $S$ is the contact area between droplet and substrate. ${ }^{48}$ In the case of sample with holes, the contact area between the droplet and the substrate is smaller than that of the sample without holes, resulting in the decline of $f_{\mathrm{v}}$ and speedup of

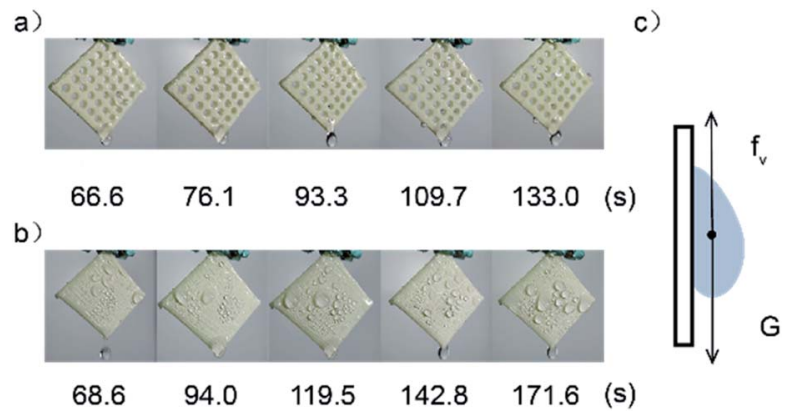

Fig. 7 Photographs of the moments of the first five droplets started to drip from the substrates with (a) and without holes (b). The corresponding time of the moments are shown below each image. (c) Schematic diagram of droplet force analysis on sample during the transportation, where $G$ represents force of gravity, and $f_{v}$ represents viscous force (see main text). droplet transportation on the substrate. Therefore, the sample with constructed parameters of mass ratio adhesives $1: 1$, cone distance $2 \mathrm{~mm}$ and printed holes was chosen in consideration of the optimized water-collection performance. The corresponding WCR (2.68 $\mathrm{g} \mathrm{h}^{-1} \mathrm{~cm}^{-2}$ ) ranks relatively highly in comparison to the reported results, especially compared with the previous reports based on a single cactus-biomimetic

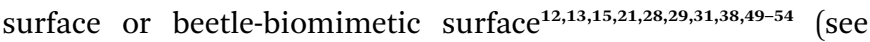
Fig. $\mathrm{S} 8 \uparrow$ for details). The similar conclusion can be drawn from our experiment (Fig. 5a), where the sample without coating exhibit lower WCR than those with coatings.

\section{Conclusion}

In summary, we have utilized UV-induced controllable diffusion and $3 \mathrm{D}$ printing method to fabricate the biomimetic materials combining beetle-like superhydrophobic-superhydrophilic character and cactus-like cone arrays, which provides good performance in water collection. The UV-induced controllable diffusion method provides a new thought of regulating hybrid wettability, which has a promising application for improving water collection efficiency. Furthermore, 3D printing technology is bound to have a wide range of applications on water collection in the future, especially the research involving geometric structure optimization. Therefore, we believe that our study provides the idea for the large-scale application of the bionic fog collector as well as the design of similar biomimetic materials.

\section{Author contributions}

The manuscript was written through contributions of all authors. All authors have given approval to the final version of the manuscript.

\section{Conflicts of interest}

There are no conflicts to declare.

\section{Acknowledgements}

This work was supported by Natural Science Foundation of China (21673243 and 11804118), Guangdong-Hong Kong Joint Innovation Project of Guangdong Province, China (2014B050505015) and Guangdong Basic and Applied Basic Research Foundation (No. 2020A1515010544).

\section{Notes and references}

1 M. M. Mekonnen and Y. A. Hoekstra, Am. Assoc. Adv. Sci., 2016, 2, 1-7.

2 M. Herrmann, Nature, 2014, 505, 10-11.

3 S. H. Schneider, Encyclopedia of climate and weather, Oxford University Press, 2011, vol. 1.

4 T. Zhao, Y. Zheng, L. Jiang, J. Ju, H. Bai and R. Fang, Nat. Commun., 2012, 3, 1246-1247. 
5 K. Li, S. Dou, L. Jiang, J. Ju, K. Liu and M. Cao, Adv. Funct. Mater., 2014, 24, 3235-3240.

6 F. Bai, J. Wu, G. Gong and L. Guo, Adv. Sci., 2015, 2, 1500047.

7 N. Desert and T. N. Desert, Nature, 2001, 414, 33-34.

8 L. Zhai, M. C. Berg, F. Ç. Cebeci, Y. Kim, J. M. Milwid, M. F. Rubner and R. E. Cohen, Nano Lett., 2006, 6, 12131217.

9 X. Wang, J. Zeng, X. Yu, C. Liang and Y. Zhang, Appl. Surf. Sci., 2019, 465, 986-994.

10 T. Nørgaard and M. Dacke, Front. Zool., 2010, 7, 1-8.

11 H. Bai, L. Wang, J. Ju, R. Sun, Y. Zheng and L. Jiang, Adv. Mater., 2014, 26, 5025-5030.

12 Y. Wang, L. Zhang, J. Wu, M. N. Hedhili and P. Wang, J. Mater. Chem. A, 2015, 3, 18963-18969.

13 L. Zhang, J. Wu, M. N. Hedhili, X. Yang and P. Wang, J. Mater. Chem. A, 2015, 3, 2844-2852.

14 Y. Xing, W. Shang, Q. Wang, S. Feng, Y. Hou and Y. Zheng, ACS Appl. Mater. Interfaces, 2019, 11, 10951-10958.

15 O. Al-Khayat, J. K. Hong, D. M. Beck, A. I. Minett and C. Neto, ACS Appl. Mater. Interfaces, 2017, 9, 13676-13684.

16 C. Luo, Langmuir, 2015, 31, 11809-11813.

17 X. Heng, M. Xiang, Z. Lu and C. Luo, ACS Appl. Mater. Interfaces, 2014, 6, 8032-8041.

18 C. Wen, H. Guo, H. Bai, T. Xu, M. Liu, J. Yang, Y. Zhu, W. Zhao, J. Zhang, M. Cao and L. Zhang, ACS Appl. Mater. Interfaces, 2019, 11, 34330-34337.

19 J. Sun, B. Bao, J. Jiang, M. He, X. Zhang and Y. Song, RSC Adv., 2016, 6, 31470-31475.

20 C. Xu, R. Feng, F. Song, X. L. Wang and Y. Z. Wang, ACS Sustainable Chem. Eng., 2018, 6, 14679-14684.

21 H. Zhu and Z. Guo, Chem. Commun., 2016, 52, 6809-6812.

22 Y. Chen, Q. Xiang, Z. Li, Y. Wang, Y. Meng and H. Duan, Nano Lett., 2016, 16, 3253-3259.

23 H. Kai, R. Toyosato and M. Nishizawa, RSC Adv., 2018, 8, 15985-15990.

24 J. Lin, X. Tan, T. Shi, Z. Tang and G. Liao, ACS Appl. Mater. Interfaces, 2018, 10, 44815-44824.

25 Y. Wang, X. Wang, C. Lai, H. Hu, Y. Kong, B. Fei and J. H. Xin, ACS Appl. Mater. Interfaces, 2016, 8, 2950-2960.

26 Y. Zhu, J. Shi, Q. Huang, L. Wang and G. Xu, Chem. Commun., 2017, 53, 2363-2366.

27 J. Ju, K. Xiao, X. Yao, H. Bai and L. Jiang, Adv. Mater., 2013, 25, 5937-5942.

28 H. Zhou, M. Zhang, C. Li, C. Gao and Y. Zheng, Small, 2018, 14, 1-7.

29 T. Xu, Y. Lin, M. Zhang, W. Shi and Y. Zheng, ACS Nano, 2016, 10, 10681-10688.

30 J. Ju, X. Yao, S. Yang, L. Wang, R. Sun, Y. He and L. Jiang, Adv. Funct. Mater., 2014, 24, 6933-6938.
31 J. Wang, S. Yi, Z. Yang, Y. Chen, L. Jiang and C. P. Wong, ACS Appl. Mater. Interfaces, 2020, 12, 21080-21087.

32 M. Wang, Q. Liu, H. Zhang, C. Wang, L. Wang, B. Xiang, Y. Fan, C. F. Guo and S. Ruan, ACS Appl. Mater. Interfaces, 2017, 9, 29248-29254.

33 Y. Yang, X. Li, X. Zheng, Z. Chen, Q. Zhou and Y. Chen, Adv. Mater., 2018, 30, 1-11.

34 X. Liu, H. Gu, M. Wang, X. Du, B. Gao, A. Elbaz, L. Sun, J. Liao, P. Xiao and Z. Gu, Adv. Mater., 2018, 30, 1-8.

35 S. Ma, M. Scaraggi, C. Yan, X. Wang, S. N. Gorb, D. Dini and F. Zhou, Small, 2019, 15, 1-13.

36 L. Xiao, G. Li, Y. Cai, Z. Cui, J. Fang, H. Cheng, Y. Zhang, T. Duan, H. Zang, H. Liu, S. Li, Z. Ni and Y. Hu, Chem. Eng. J., 2020, 399, 125139.

37 X. Li, Y. Yang, L. Liu, Y. Chen, M. Chu, H. Sun, W. Shan and Y. Chen, Adv. Mater. Interfaces, 2020, 7, 1-10.

38 H. Bai, C. Zhang, Z. Long, H. Geng, T. Ba, Y. Fan, C. Yu, K. Li, M. Cao and L. Jiang, J. Mater. Chem. A, 2018, 6, 20966-20972.

39 R. Hu, N. Wang, L. Hou, Z. Cui, J. Liu, D. Li, Q. Li, H. Zhang and Y. Zhao, J. Mater. Chem. A, 2019, 7, 124-132.

40 J. Hou, Q. Shi, W. Ye, P. Stagnaro and J. Yin, Chem. Commun., 2014, 50, 14975-14978.

41 J. F. Moulder, W. F. Stickle, P. E. Sobol and K. D. Bomben, Handbook of X-Ray Photoelectron Spectroscopy, ed. J. Chastain, Perkin-Elmer Corporation, Eden Prairie, MN, 1992.

42 S. Pazokifard, S. M. Mirabedini, M. Esfandeh and S. Farrokhpay, Adv. Powder Technol., 2012, 23, 428-436.

43 J. Yu, H. Yu, B. Cheng, M. Zhou and X. Zhao, J. Mol. Catal. A: Chem., 2006, 253, 112-118.

44 Y. Huang, Y. Hu, C. Zhu, F. Zhang, H. Li, X. Lu and S. Meng, Adv. Mater. Interfaces, 2016, 3, 1-5.

45 É. Lorenceau and D. Quéré, J. Fluid Mech., 2004, 510, 29-45.

46 D. Seo, J. Lee, C. Lee and Y. Nam, Sci. Rep., 2016, 6, 1-11.

47 S. Yang, N. Sun, X. Dai, S. O. Nielsen, B. B. Stogin, T.-S. Wong and J. Wang, Sci. Adv., 2018, 4, eaaq0919.

48 A. Mohammad Karim and H. P. Kavehpour, Colloids Surf., A, 2018, 548, 54-60.

49 B. Wang, Y. Zhang, W. Liang, G. Wang, Z. Guo and W. Liu, J. Mater. Chem. A, 2014, 2, 7845-7852.

50 X. Wang, J. Zeng, X. Yu and Y. Zhang, J. Mater. Chem. A, 2019, 7, 5426-5433.

51 Y. Peng, Y. He, S. Yang, S. Ben, M. Cao, K. Li, K. Liu and L. Jiang, Adv. Funct. Mater., 2015, 25, 5967-5971.

52 J.-A. Duan, H. Du, J. He, K. Yin, C. Wang and X. Dong, Nanoscale, 2017, 9, 14620-14626.

53 H. Zhu, R. Duan, X. Wang, J. Yang, J. Wang, Y. Huang and F. Xia, Nanoscale, 2018, 10, 13045-13054.

54 L. Zhong, J. Feng and Z. Guo, J. Mater. Chem. A, 2019, 7, 8405-8413. 